\title{
WHIRLING MOTIONS OF A BEAM GENERATED BY IN-PLANE PULSES
}

\author{
A. Behboud AND R. A. Scott \\ Department of Mechanical Engineering and Applied Mechanics, The University of Michigan, \\ Ann Arbor, Michigan 48109-2121, U.S.A.
}

(Received 15 October 1990 and in final form 27 March 1991)

\section{INTRODUCTION}

An extensive body of literature now exists on the instability to out-of-plane perturbations of beams forced harmonically in a plane, i.e., whirling motion. An early study on nonlinear beam dynamics for whirling motions can be found in Ho, Scott and Eisley [1], who

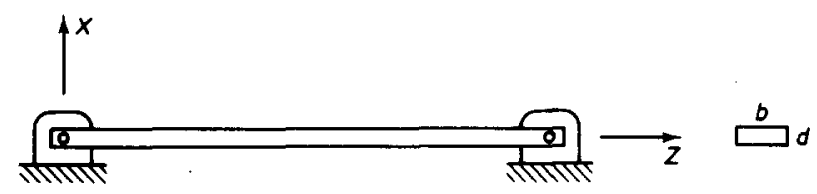

Figure 1. Beam geometry.

investigated motions of compact beams with pinned ends. They used a model in which extensional effects were included, but non-linear curvature and longitudinal inertia were neglected. Since then, more accurate mechanical modeling has been developed. Venkatesen and Nagaraj [2] derived a model in which all three effects were included, but in their results an inextensionality condition was assumed (in view of the fact that the beam was a cantilever). Such a condition was also assumed by Nayfeh and Pai [3], who analyzed threedimensional, integro-differential equations of motion for a cantilever beam, longitudinal inertia and non-linear curvature being included. Extensive work in the field has also been done by Crespo da Silva. In recent contributions [4,5], longitudinal inertia, non-linear curvature, extension and torsion were included in his three-dimensional equations of motion.

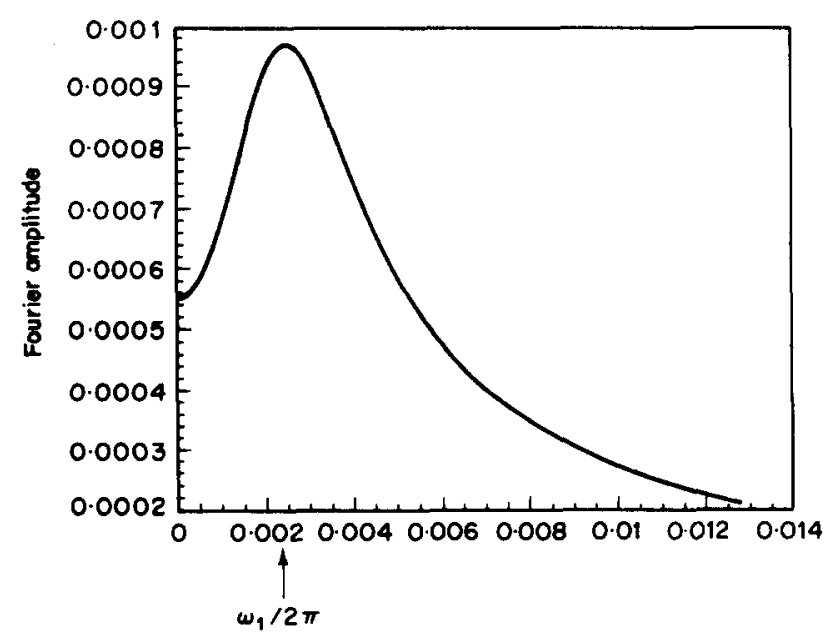

Figure 2. The spectrum of the pulse for $F_{x}=2.5 \times 10^{-6}, \omega_{1} / \omega_{n}=1$ and $q=0.01$. 
In the above works the forcing functions were harmonic and of primary concern were frequency-amplitude relations and associated stability questions. To the authors' knowledge, no studies have addressed the issue of possible out-of-plane instabilities of beams subject to plane, pulse loading. It is the central question addressed here for a pinnedpinned beam.

\section{EQUATIONS OF MOTION}

Ho, Scott and Eisley [1] developed a set of equations for the non-linear, whirling motions of compact beams, in which the source of the non-linearity was centerline extension. In their model, non-linear curvature and longitudinal inertia were neglected.

For pinned-pinned beams it has recently been shown [6], using Galerkin's method and numerical integration in time, that under a broad range of circumstances non-linear curvature played an insignificant role in the response to planar, harmonic forcing. Consequently it is neglected here. Retention of longitudinal inertia and centerline extension
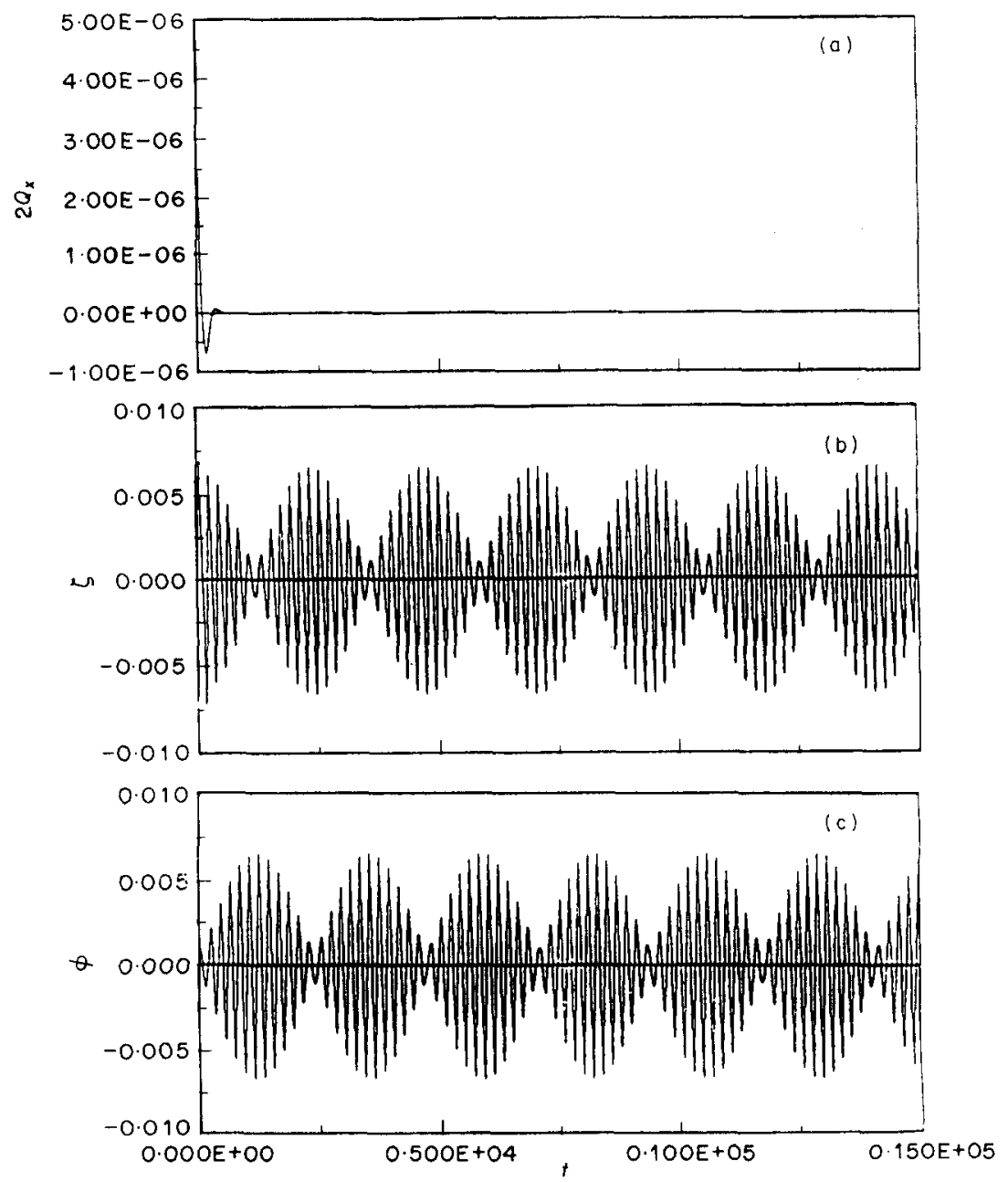

Figure 3. Time histories of (a) the forcing function $2 Q_{x}$, (b) the $x$-component of displacement, and (c) the $y$-component of displacement for $F_{x}=2.5 \times 10^{-6}, \omega_{1}=\omega_{n}, q=0.01$ and zero damping: square beam. 
was found to be important. Retaining longitidinal inertia, Ho, Scott and Eisley's equations become:

$$
\begin{gathered}
\left(I_{y y} / A L^{2}\right) u^{\prime \prime \prime}-\frac{1}{2}\left[3 u^{\prime 2} u^{\prime \prime}+u^{\prime \prime} v^{\prime 2}+2 u^{\prime} v^{\prime} v^{\prime \prime}+2 w^{\prime \prime} w^{\prime}+2 u^{\prime} w^{\prime \prime}\right]+\ddot{u}=(L / E A) f_{x}(s, \tau), \\
\left(I_{x x} / A L^{2}\right) v^{\prime \prime \prime}-\frac{1}{2}\left[3 v^{\prime 2} v^{\prime \prime}+v^{\prime \prime} u^{\prime 2}+2 v^{\prime} u^{\prime} u^{\prime \prime}+2 w^{\prime \prime} w^{\prime}+2 v^{\prime} w^{\prime \prime}\right]+\ddot{v}=0 \\
w^{\prime \prime}+u^{\prime} u^{\prime \prime}+v^{\prime} v^{\prime \prime}-\ddot{w}=0 .
\end{gathered}
$$

The basic configuration of the beam is shown in Figure $1: u, v$ and $w$ are the displacement components in the $x, y$ and $z$ directions, respectively. A prime denotes differentiation with respect to the dimensionless variable $s \equiv z / L$, and a dot differentiation with respect to the dimensionless time $\tau=(t / L) \sqrt{E / \rho}, E$ being Young's modulus and $\rho$ being the mass density. $A$ is the cross-sectional area and $I_{x x}, I_{y y}$ are area moments of inertia. The beam is being forced in the $x-z$ plane in the $x$-direction only, with a forcing function given by $f_{x}(s, \tau)$.

Galerkin's method is now employed to obtain ordinary differential equations in time. A one-mode approximation is used; namely,

$$
u=\zeta(\tau) \sin \pi s, \quad v=\phi(\tau) \sin \pi s, \quad w=\eta(\tau) \sin 2 \pi s .
$$

Note the factor of 2 in equation (6), reflecting the fact that, as the longitudinal motion goes through one cycle, the transverse motion goes through two. Introducing equations (4)-(6) into equations (1)-(3), multiplying equations (1)-(3) by $\zeta, \phi$ and $\eta$, respectively, and integrating from $s=0$ to $s=1$, gives

$$
\begin{gathered}
\ddot{\zeta}=-\left(I_{y y} / A L^{2}\right) \pi^{4} \zeta-(3 / 8) \pi^{4} \zeta^{3}-(3 / 8) \pi^{4} \zeta \varphi^{2}-\pi^{3} \zeta \eta+2 Q_{x}, \\
\ddot{\phi}=-\left(I_{x x} / A L^{2}\right) \pi^{4} \phi-(3 / 8) \pi^{4} \phi^{3}-(3 / 8) \pi^{4} \phi \zeta^{2}-\pi^{3} \phi \eta, \\
\ddot{\eta}=-4 \pi^{2} \eta-\left(\pi^{3} / 2\right) \zeta^{2}-\left(\pi^{3} / 2\right) \phi^{2},
\end{gathered}
$$

where

$$
Q_{x}(\tau)=(L / E A) \int_{0}^{1} f_{x}(s, \tau) \sin \pi s \mathrm{~d} s .
$$

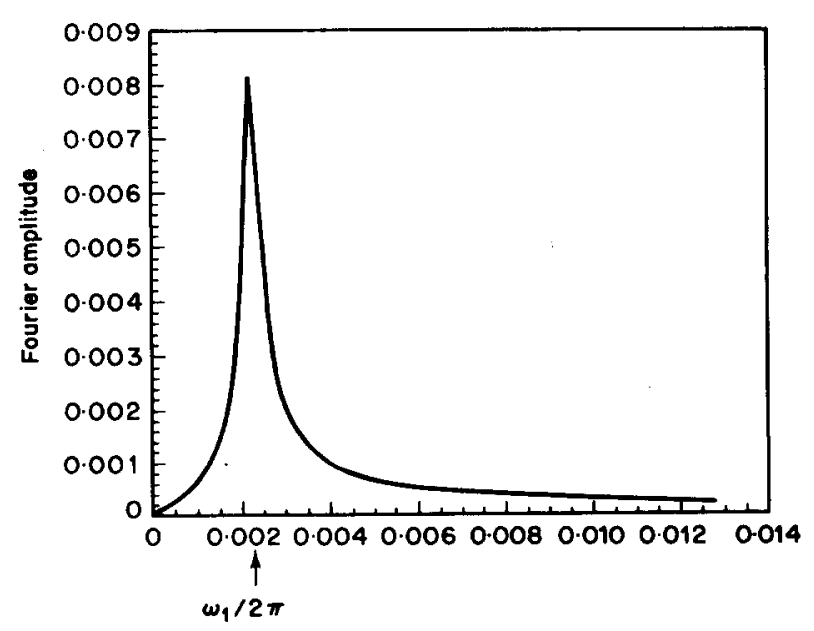

Figure 4. The spectrum of the pulse for $F_{x}=2.5 \times 10^{-6}, \omega_{1}=\omega_{n}$ and $q=0.001$. 


\section{NUMERICAL SIMULATIONS}

The fundamental question addressed is whether a plane pulse, depending on its frequency spectrum, could induce out-of-plane motions. The spatial distribution of the pulse was taken to be a constant, $F_{0}$. The time dependence was taken to be a decaying cosinusoid, so that

$$
Q_{x}(\tau)=F_{x} \mathrm{e}^{-q \tau} \cos \omega_{1} \tau,
$$

where $F_{x}=\left(2 L F_{0}\right) /(\pi E A)$. As shown in Harris and Crede [7], the spectrum of this pulse is concentrated around the frequency $\omega_{1}$ and its width in the frequency domain increases as $q$ decreases. Of primary interest is whether a pulse the spectrum of which is centered around a frequency known to cause whirling motions for harmonic forcing would also generate three-dimensional motion. Conversely, if the spectrum is centered around a frequency which is stable (in the harmonic case) to out-of-plane-perturbations, the question is if only in-plane motions would occur.

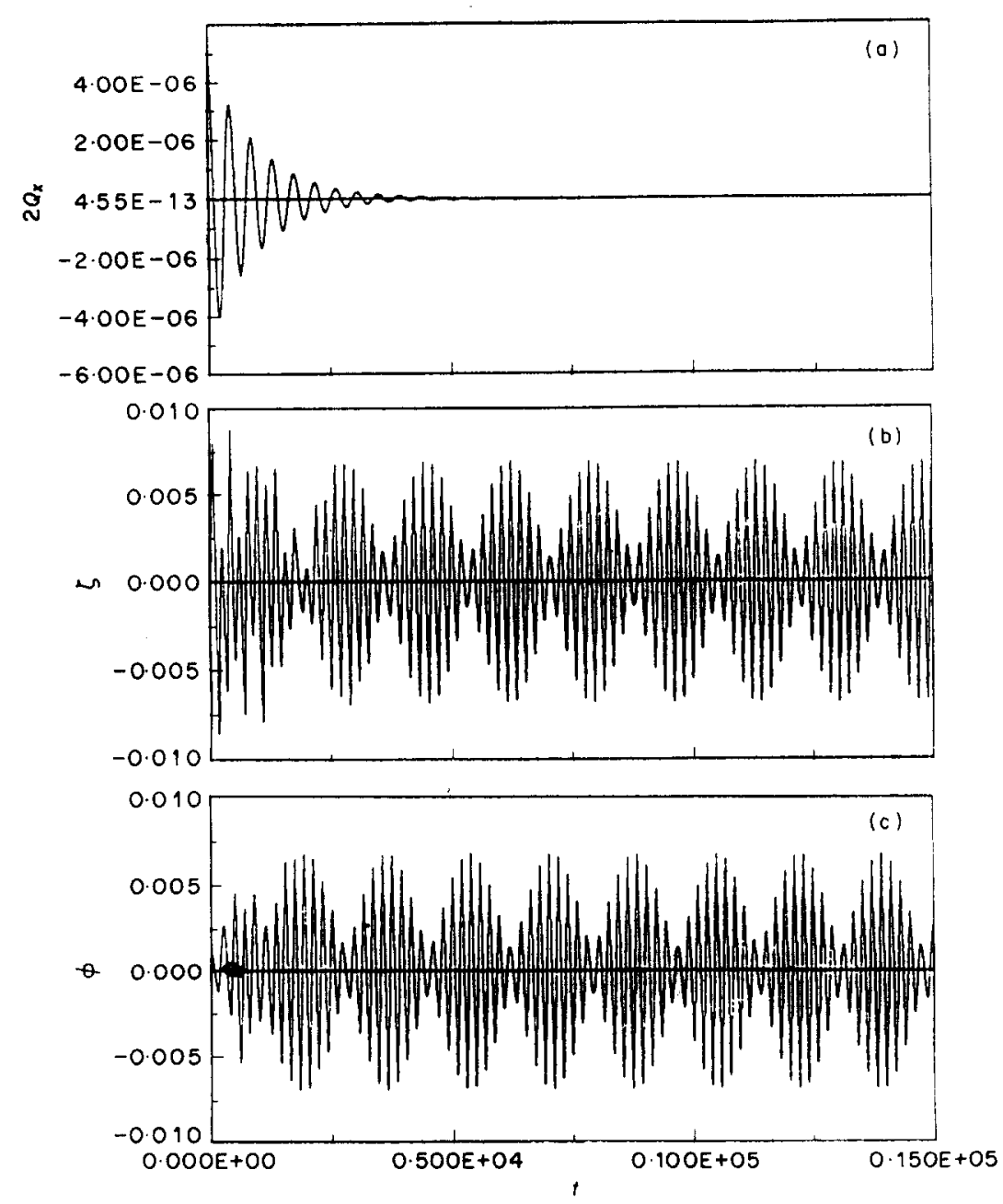

Figure 5. Time histories of (a) the forcing function $2 Q_{x}$, (b) the $x$-component, and (c) the $y$-component of displacement for $F_{x}=2 \cdot 5 \times 10^{-6}, \omega_{1}=\omega_{n}, q=0.001$ and zero damping: square beam. 

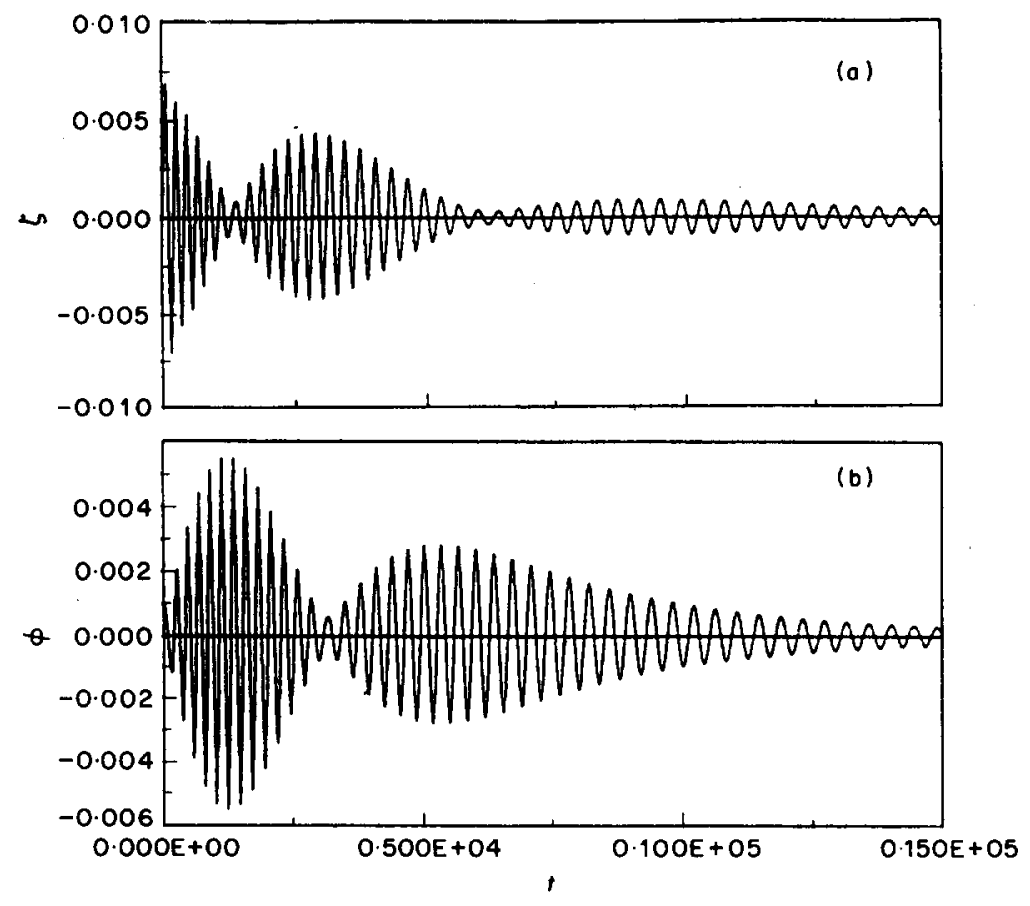

Figure 6. Time history of (a) the $x$-component of displacement, and (b) the $y$-component of displacement for $F_{x}=2.5 \times 10^{-6}, \omega_{1}=\omega_{n}, q=0.01$ and $1.35 \%$ damping: square beam.

The ordinary differential equations (7)-(9) were integrated using a Fehlberg fourth-fifth order Runge-Kutta method available from the NAAS Library (routine DRKF45). In all cases, the simulation is started with a very small out-of-plane component. By whirling motion is meant motions which grow from the initial small level. Consider first the case of a square beam $\left(I_{x x}=I_{y y}\right)$, with the slenderness ratio $d / L=0.005$ inches (as in Ho, Scott and Eisley [1]), and zero damping. Also $F_{x}=2.5 \times 10^{-6}, q=0.01$ and $\omega_{1}=\omega_{n}$, where $\omega_{n}$ is the first natural non-dimensional bending frequency (in the linear case). The Fourier amplitude spectrum of the pulse is shown in Figure 2. It is quite broad and spans frequencies that in the harmonic case would lead Ho et al. [1] to predict out-of-plane motions. Shown in Figures $3(\mathrm{a}-\mathrm{c})$ are the time histories of the force and the $x$ and $y$ components of displacement. The motion is indeed whirling. After the initial transient, a beating motion between the two planes occurs.

A pulse with $F_{x}=2.5 \times 10^{-6}, \omega_{1}=\omega_{n}, q=0.001$ is now applied: the spectrum is shown in Figure 4, and is strongly localized around $\omega_{1}$. At this frequency and force level Ho et al. [1] would predict in-plane motions and one could anticipate, perhaps naively, that the pulse would produce in-plane motions. The time histories of the force and displacements are shown in Figures $5(\mathrm{a}-\mathrm{c})$. Somewhat surprisingly, the motion is still three-dimensional (different from the previous case in that the beating motions are faster).

The role of damping (which is always present to some degree and is artificially injected here into the equations of motion using a viscous damping model) was also addressed. Consider the case of $1.35 \%$ damping, $F_{x}=2.5 \times 10^{-6}, \omega_{1}=\omega_{n}$ and $q=0.01$. The time histories of the displacements are given in Figures 6(a) and (b). As can be seen from Figure $6(\mathrm{~b})$, there is considerable growth of the initial $y$-motion before damping quenches it. Whirling motions still occur. 


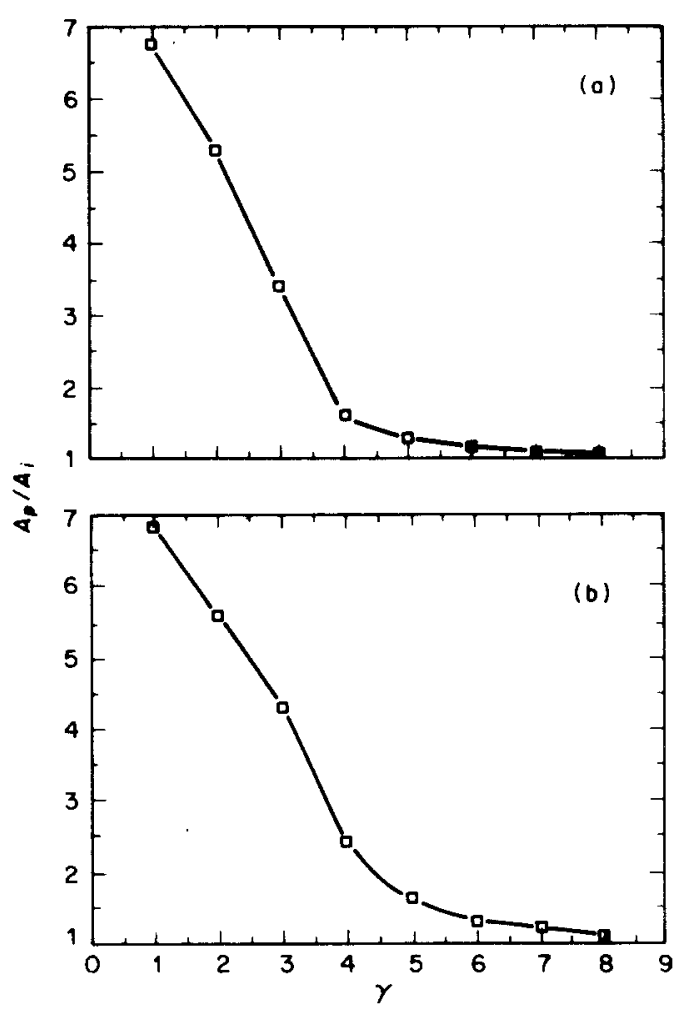

Figure 7. Peak values $A_{p}$ as a function of aspect ratio $\gamma=b / d$ for (a) $q=0.01$ and (b) $q=0.001$.

Non-square beams were then examined. Beating motions were found in all cases studied, but fulltime histories will not be given here. Shown in Figures 7(a) and 7(b) are plots of $A_{p} / A_{i}$ versus $\gamma$, where $A_{p}$ and $A_{i}$ are the peak and initial values, respectively, of the outof-plane response and $\gamma=b / d$ (see Figure 1). In Figure $7(\mathrm{a}), q=0 \cdot 01$, and in Figure 7(b), $q=0.001$. In both cases $F_{x}=2.5 \times 10^{-6}, \omega_{1}=\omega_{n}$, and zero damping was assumed. From the figures it is seen that, for all $\gamma$ values, the more localized pulse $(q=0.001)$ generates larger amplitude out-of-plane motions than the diffuse one $(q=0.01)$. No clear-cut pattern emerges, but the broad conclusion may be reached that for a wide range of parameters, and to some degree or another, in-plane pulses always induce whirling motions.

\section{REFERENCES}

1. C. H. Ho, R. A. SCOTT and J. G. ErsL.Ey 1975 International Journal of Nonlinear Mechanics 10, 113-127. Non-planar, nonlinear oscillations of a beam.

2. C. Venkatesan and V. T. NAgaraj 1982 Journal of Sound and Vibration 84, 549-556. Nonlinear flapping vibrations of rotating blades.

3. A. H. NAYFEH and F. F. PAI 1989 International Journal of Nonlinear Mechanics 24, 139-158. Nonlinear non-planar parametric responses of an inextensional beam.

4. M. R. M. CReSPo DA Silva 1988 International Journal of Solids and Structures 24, 1225-1234. Non-linear flexural-flexural-torsional-extensional dynamics of beams---I: Formulation.

5. M. R. M. CRespo Da Silva 1988 International Journal of Solids and Structures 24, 1235-1242. Non-linear flexural-flexural-torsional-extensional dynamics of beams-II : Response analysis.

6. A. Behboud 1990 Ph.D. Dissertation, University of Michigan. An analytical and numerical investigation on nonlinear beam dynamics.

7. C. M. HARRis and C. E. CReDE (editors) 1976 Shock and Vibration Handbook. New York: McGraw-Hill. 